\title{
Two-point resolution of asymmetrically apodized optical systems
}

\author{
Andra Naresh Kumar Reddy(1,2,*), Dasari Karuna Sagar(3) \\ 1. Department of Physics, University College of Science, Osmania University, Hyderabad-500007, India. \\ 2. Department of Humanities \& Sciences, KG Reddy College of Engineering \& Technology, Chilkur (v), Moinabad (M), \\ R.R. District - 501 504, Andhra Pradesh (State), India. \\ 3. Department of Physics, Nizam College, Osmania University, Hyderabad-500001, India. \\ (") Email: naarereddy@osmania.ac.in \\ Received / Recibido: 26/11/2012. Revised / Revisado: 13/05/2013. Accepted / Aceptado: 17/05/2013. \\ DOI: http://dx.doi.org/10.7149/OPA.46.3.215
}

\begin{abstract}
:
Effect of asymmetric apodization applied to two dimensional complex pupil filters on resolution of object points in the image plane of apodized optical systems has been investigated. The required pupil function and its transmittance is formulated and studied. These sparrow limits have been discussed as functions of the degree of spatial coherence of the illumination, intensity ratio of two object points and asymmetric apodization parameter $(b)$. The sparrow resolution limit increases as the degree of coherence increases, irrespective of the intensity ratio of two point objects. With respect to intensity ratio of two points it is clearly found that for all cases of illumination, employing complex pupil filters in the presence of asymmetric apodization leads to an improvement in resolution of two object points.
\end{abstract}

Key words: Asymmetric Apodization, Complex-Pupil Filter, Sparrow Resolution Limit, Superresolution, Two-Point Resolution, Degree of Coherence, Intensity Ratio.

\section{REFERENCES AND LINKS / REFERENCIAS Y ENLACES}

[1]. P. Jacquinot, B. Roizen-Dossier B, “Apodisation”, Prog. Optics 3, 29-132 (1964). DOI

[2]. B. R. Frieden, "Evaluation, design and extrapolation methods for optical signals, based on use of the Prolate functions", Prog. Optics 9, 311-407 (1971). DOI

[3]. J. Ojeda-Castaneda, L. R. Berriel-Valdos, E, Montes, "Spatial filter for increasing the depth of focus", Opt. Lett. 10, 520-522 (1985). DOI

[4]. R. Barakat, "Solution of the Luneberg apodization problems", J. Opt. Soc. Am. 52, 264-272 (1962). DOI

[5]. L.Cheng, G. G. Siu, "Asymmetric apodization", Meas. Sci. Technol. 2, 198-202 (1991). DOI

[6]. G. G. Siu, L. Cheng, D. S. Chiu, K. S. Chang, "Improved side-lobe suppression in asymmetric apodization", J. Phys. D: Appl. Phys. 27, 459-463 (1994). DOI

[7]. T Asakura, "Resolution of two unequally bright points with partially coherent light", Nouv. Rev. Opt. 5, 169-177 (1974). DOI

[8]. T Asakura, T. Ueno, "Apodization for increasing two-point resolution by the sparrow criterion under the partially coherent illumination", Nouv. Rev. Opt. 5, 349-359 (1974). DOI

[9]. R. Barakat, "Application of apodization to increase two-point resolution by Sparrow criterion. Icoherent illumination", J. Opt. Soc. Am. 52, 276-283 (1962). DOI

[10]. M. Kowalczyk, C. J. Zapata-Rodriguez, M. Martinez-Corral, "Asymmetric apodization in confocal scanning systems", Appl. Opt. 37, 8206-8214 (1998). DOI

[11].M. Kowalczyk, P. Andres, C. J. Zapata-Rodriguez, M. Martinez-Corral, "Three-dimensional superresolution by annular binary filters", Opt. Commun. 165, 267-278 (1999). DOI

[12]. T. Cichocki, M. Martinez-Corral, M. Kowalczyk, P. Andres, "Annular binary filters for controlling the axial behaviour of optical systems", J. Mod. Opt. 45, 227-237(1998). DOI 
[13]. M. Kowalczyk, C. Zapata, E. Silvestre, M. Martinez-Corral, "Phase pupil filters for improvement of the axial resolution in confocal scanning microscopy”, Opt. Appl. 28, 128-137(1998).

[14]. W. Yang, A. B. Kostinski, "One-sided achromatic phase apodization for imaging of extrasolar planets", Astroph. J. Lett. 605, 892-901 (2004). DOI

\section{Introduction}

Apodization defines that the process of the suppressing of the side lobes of diffraction feet so as to improve resolution of optical system for two coherent or incoherent object points separated by different distance. It is most widely studied classical problem in wave optics. An excellent review on apodization technique has been investigated by Jacquinot and RoizenDossier [1]. Frieden [2] introduced possible way of making central peak of diffracted point spread function as narrow as possible and to achieve sidelobe suppression as low as possible. OjedaCastaneda et al. [3] investigated axial apodization of rotationally symmetric apodized optical systems. Barakat [4] investigated the energy enclosed in first dark ring of radius in the feet of diffraction pattern. All these studies belong to the category of symmetric apodization in which suppression of sidelobes is achieved at the cost of degrading central maxima. Cheng and Siu [5] introduced asymmetric apodization and succeed in achieving low side-lobes and steep central peak on one side termed as 'good side' at the cost of worsening its counterpart, the 'bad side' which has fairly high side-lobes and broad central peak. In further continuation of their work [6] they obtained improved side-lobe suppression. Their works can be considered as a significant breakthrough in apodization studies.

Asakura [7] investigated resolution of two unequally bright point objects with partial coherent illumination. Asakura et al. [8] observed effect of apodization on two point resolution by sparrow criterion. Symmetric apodization improves resolution of two point objects by sparrow criterion under incoherent illumination [9]. These studies are the basis for our investigation. In this work we have investigated asymmetric apodization which aims to improve resolution of composite image of optical system containing a faint object which is in close proximity of bright object or two point objects with equal intensity. This can be achieved by introducing suitable apodizers in the optical systems as complex pupil filters in the presence asymmetric apodization. Many methods have been developed for design of apodizers to achieve superresolution based on both amplitude-phase profiles. The study of asymmetric apodization found many applications in different potential fields of image processing, confocal microscopy, spectroscopy, medical imaging, communication and micro electronics. For instance set up of asymmetric apodization circular plate in spectrometer or confocal microscopy system facilitates to detect the presence of weak image point in close proximity of bright image point. Kowalczyk et al. [10] demonstrated application of asymmetric apodization to achieve superresolution in confocal scanning systems. Later [11] they have presented annular binary filters for improving three-dimensional resolving power of optical systems. In extension to their work [11] they applied annular binary pupil filters for controlling the axial resolution of optical systems. Kowalczyk et al. [13] have proposed Phase-only pupil filters for asymmetric axial resolution of point spread function. Yang and Kostinski [14] applied the same technique to detect extra solar planets.

\section{Theory}

The expression for composite image intensity distribution in image plane of an apodized optical system, as a function of reduced coordinate $Z$, is given by:

$$
\begin{aligned}
I(Z)=\mid & \left.G(Z-B)\right|^{2}+\alpha|G(Z+B)|^{2}+ \\
& +2 \sqrt{\alpha} \gamma\left(Z_{0}\right)|G(Z+B)||G(Z-B)|,
\end{aligned}
$$

where $Z_{0}$ is the actual separation between the object points, $\alpha$ is the ratio of their intensities and $\gamma\left(Z_{0}\right)$ is the real part of the complex degree of spatial coherence of the illumination. $Z$ is the dimension less diffraction variable. $G(Z+B)$ and $G(Z-B)$ are the normalized complex amplitude impulse response functions of the optical imaging system corresponding to the 
object points, each of which is situated at a distance of $Z_{0} / 2$ on either side of the optical axis. The amplitude impulse response functions $G(Z \pm B)$ are given by:

$$
G(Z \pm B)=2 \int_{0}^{1} J_{0}[(Z \pm B) r] r d r
$$

where $J_{0}$ is the Bessel function of first kind and zero order, and $r$ is the normalized distance of a general point on the exit pupil varying from 0 to 1. In the present case, as the shaded aperture amplitude and phase filter is used for asymmetrically apodising the optical system, the above Eqs. (2) become:

$$
G(Z \pm B)=2 \int_{0}^{1} f(r) J_{0}[(Z \pm B) r] r d r
$$

where $f(r)$ is phase and amplitude complex pupil function and is given by:

$$
f(r)=\left\{\begin{array}{cc}
-i e^{i u r \cos (\phi-\varphi)} & 1-b \leq r \leq 1, \\
& -\frac{\pi}{2} \leq \varphi \leq \frac{\pi}{2} \\
t(r) e^{i u r \cos (\phi-\varphi)} & 0 \leq r \leq 1-b, \\
& 0 \leq \varphi \leq 2 \pi \\
i e^{i u r \cos (\phi-\varphi)} & 1-b \leq r \leq 1 \\
& \frac{\pi}{2} \leq \varphi \leq \frac{3 \pi}{2} .
\end{array}\right.
$$

It is clear that for $t(r)=1$, gives uniform amplitude transmittance over the central region of the pupil. Because of exceptionally deep reduction ability and constant working angles through out the regions of considered semicircular edge rings, we consider the odd (antisymmetric) phase functions.

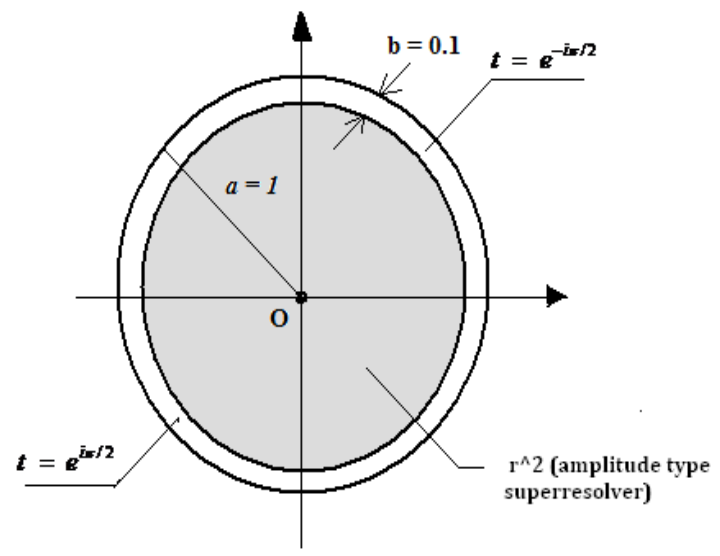

Fig. 1. Design of asymmetric complex pupil filters.
If $t(r)=r^{2}$, the parabolic filters are the super-resolving type of filters for which amplitude transmittance increases from the centre to edge of the pupil, where $r$ is the normalized distance of a point on the exit pupil varying from 0 to 1

For asymmetrically apodized circular apertures, the expression derived for complex amplitude impulse response is given by:

$$
\begin{aligned}
& G(Z \pm B)= \\
& =2 \int_{0}^{-\delta}\left[\int_{0}^{1-b} f(r) J_{0}(Z \pm B) r d r\right] Z d Z+ \\
& +i \int_{1-b \frac{\pi}{2}}^{1} \int^{\frac{3 \pi}{2}} e^{i u r \cos (\phi-\varphi)} r d r d \varphi- \\
& -i \int_{1-b \frac{-\pi}{2}}^{1} \int^{\frac{\pi}{2}} e^{i u r \cos (\phi-\varphi)} r d r d \varphi .
\end{aligned}
$$

In presence of asymmetric apodization, the expression for total image intensity distribution is obtained with substitution of Eq. (5) in Eq. (1), where $r$ is the coordinate in pupil plane, $u$ is the dimensionless diffraction coordinate in image plane and $f(r)$ is the pupil function of optical system.

The modified Sparrow criterion introduced by Asakura [8] states that, "the resolution is retained when the second derivative of the image intensity distribution vanishes at a certain point $\left(Z=Z_{0}^{1}\right)$ between two Gaussian image points, with the condition that this point $Z_{0}^{1}$ should be a solution for the first derivative of the image intensity distribution becoming zero". This can be expressed mathematically as:

$$
\begin{gathered}
\left|\frac{\partial^{2} I(Z)}{\partial Z^{2}}\right|_{Z=Z_{0}^{1}}=0 \\
\left|\frac{\partial I(Z)}{\partial Z^{2}}\right|_{Z=Z_{0}^{1}}=0 .
\end{gathered}
$$

\section{Results and discussion}

Aim to improve resolution of two point objects with coherent or partially light we proposed theoretical design of asymmetric complex pupil 
filters by adopting procedure as mentioned in previous section. In order to investigate effect of asymmetric apodization on the intensity distributions in the composite image of twoobject points formed by apodized optical imaging system have been obtained from Eq.(1) as function of diffraction parameter $Z$ varying from -12 to +12 by employing twelve point gauss quadrature numerical method of integration. An iterative method has been developed and applied to find the sparrow limit $\delta_{S}=Z_{0}^{1}$. It should be noted that as the limit of resolution $\delta \mathrm{s}$ increases, the overall optical resolution decreases.

Table I lists the sparrow limits of resolution as function of semi-circular edge ring width $(b)$ for various values of the intensity ratio $\alpha$ and degree of coherence $\gamma$. These values are obtained

TABLE I

Sparrow limit $\left(Z_{0}\right)$ for different amount of apodization parameter $(b)$, intensity ratio $(\alpha)$ and degree of coherence $(\gamma)$.

\begin{tabular}{|c|c|c|c|c|c|c|c|}
\hline & & \multicolumn{6}{|c|}{ Sparrow limits } \\
\hline$\alpha$ & $\gamma$ & $b=0$ & $b=0.02$ & $b=0.04$ & $b=0.06$ & $b=0.08$ & $b=0.1$ \\
\hline \multirow[t]{6}{*}{0.2} & 0 & 3.829 & 4.1216 & 4.3341 & 4.4011 & 4.3089 & 3.1441 \\
\hline & 0.2 & 4.204 & 4.4670 & 4.6328 & 4.6383 & 4.4873 & 3.4839 \\
\hline & 0.4 & 4.548 & 4.7795 & 4.9010 & 4.8438 & 4.6323 & 3.8585 \\
\hline & 0.6 & 4.859 & 5.0641 & 5.1454 & 5.0246 & 4.7504 & 4.2944 \\
\hline & 0.8 & 5.091 & 5.3070 & 5.3708 & 5.1849 & 4.8503 & 4.5404 \\
\hline & 1 & 5.110 & 5.4731 & 5.5766 & 5.3271 & 4.9336 & 4.5989 \\
\hline \multirow[t]{6}{*}{0.4} & 0 & 3.697 & 3.8629 & 3.9373 & 3.8936 & 3.7706 & 3.6290 \\
\hline & 0.2 & 4.045 & 4.1847 & 4.2165 & 4.1212 & 3.9548 & 3.7900 \\
\hline & 0.4 & 4.369 & 4.4827 & 4.4684 & 4.3158 & 4.1044 & 3.9201 \\
\hline & 0.6 & 4.673 & 4.7621 & 4.6996 & 4.4820 & 4.2250 & 4.0216 \\
\hline & 0.8 & 4.939 & 5.0220 & 4.9145 & 4.6227 & 4.3147 & 4.1062 \\
\hline & 1 & 5.108 & 5.2528 & 5.1153 & 4.7319 & 4.3824 & 4.1778 \\
\hline \multirow[t]{6}{*}{0.6} & 0 & 3.516 & 3.6060 & 3.5974 & 3.4976 & 3.3618 & 3.2656 \\
\hline & 0.2 & 3.854 & 3.9154 & 3.8603 & 3.7045 & 3.5382 & 3.4420 \\
\hline & 0.4 & 4.174 & 4.2038 & 4.0911 & 3.8659 & 3.6855 & 3.5879 \\
\hline & 0.6 & 4.480 & 4.4773 & 4.2954 & 3.9930 & 3.8102 & 3.7102 \\
\hline & 0.8 & 4.766 & 4.7407 & 4.4657 & 4.1026 & 3.9168 & 3.8140 \\
\hline & 1 & 5.004 & 4.9964 & 4.5739 & 4.1980 & 4.0088 & 3.9030 \\
\hline \multirow[t]{6}{*}{0.8} & 0 & 3.311 & 3.3248 & 3.2222 & 3.0972 & 3.0468 & 3.0330 \\
\hline & 0.2 & 3.6434 & 3.6159 & 3.4296 & 3.2948 & 3.2383 & 3.2195 \\
\hline & 0.4 & 3.9614 & 3.8852 & 3.6097 & 3.4638 & 3.4000 & 3.3754 \\
\hline & 0.6 & 4.272 & 4.1327 & 3.7682 & 3.6099 & 3.5383 & 3.5073 \\
\hline & 0.8 & 4.5747 & 4.3398 & 3.9088 & 3.7375 & 3.6578 & 3.6204 \\
\hline & 1 & 4.859 & 4.5008 & 4.0345 & 3.8498 & 3.7619 & 3.7182 \\
\hline \multirow[t]{6}{*}{1} & 0 & 2.979 & 2.8047 & 2.7842 & 2.8008 & 2.8328 & 2.8736 \\
\hline & 0.2 & 3.3064 & 3.0499 & 3.0047 & 3.0081 & 3.0310 & 3.0641 \\
\hline & 0.4 & 3.6243 & 3.2707 & 3.1977 & 3.1867 & 3.1995 & 3.2243 \\
\hline & 0.6 & 3.9402 & 3.4719 & 3.3687 & 3.3423 & 3.3446 & 3.3610 \\
\hline & 0.8 & 4.2622 & 3.6570 & 3.5215 & 3.4792 & 3.4710 & 3.4790 \\
\hline & 1 & 4.6011 & 3.8288 & 3.6590 & 3.6007 & 3.5819 & 3.5818 \\
\hline
\end{tabular}


for unapodized case $(b=0)$ and asymmetric apodized case with transparent central region $(b \neq 0)$. For widely varying intensities of two points with $\alpha=0.2$, the limit increases from 3.829 to 4.401 as the semi-circular ring width increases from 0 to 0.06 and then it reduces to 3.144 as the ring width increases to 0.1 when the illumination is incoherent, consequently, increasing the resolution of optical system. Similar trend is noticed for $\gamma=0.2$ with intensity difference $\alpha=0.2$. But for the partially coherent and coherent illumination, the limit increases as the semi-circular ring width increases from 0 to 0.04 and it is observed to decrease with further increased semi-circular ring width. Similar trend is noticed for $\alpha=0.4$.

For $\alpha=0.6$ and 0.8 , the sparrow limit increases as b increases from 0 to 0.02 and then it decreases as the semi-circular ring width increases to 0.1 . This implies that as the intensities of two object points approach 0.8 the sparrow limit decreases with semicircular ring width for incoherent, partially coherent and coherent illumination, there by increasing overall resolution effectively.

For equally bright object points with degree of incoherence, the sparrow limit decreases from 2.979 to 2.784 as semi-circular ring width goes up from 0 to 0.04 and then it rises to 2.873 as $b$ increases to 0.1 . For equal intensity object points with degree of coherence, the sparrow limit decreases from 4.6011 to 3.5818 as semicircular ring width $(b)$ increases to 0.1 . This implies that for equally bright points, as degree of asymmetric apodization increases, resolution limits obtained are lower than that of the unapodized case (Airy) irrespective of the condition of the illumination.

Figure 2 shows the intensity distribution $I(Z)$ as function of optical coordinate $(Z)$ for different amount of apodization parameter $b$. (a), (b) of this figure correspond to the cases of different transmittances in central region of pupil function. In this case, the degree of spatial coherence for the illumination is fixed at 0 and the intensity ratio of the two object points is fixed at 0.6 and the point separation $Z_{0}=4$. Figure 2(a) shows that as asymmetric apodisation $(b)$ increases the intensity profile of the incoherent composite image of two object points on left half axis become broadened and shifted while on right half axis the intensity profile becomes narrower. This simultaneous effect is a clear evidence for superresolution phenomenon. Figure 2(b) shows the effect of superresolver on the intensity profile of incoherent composite image of two unequal bright object points where for all values of apodization parameter $b$, the degree of superresolution increases fair enough.

Figure 3 gives the profile of the intensity distribution of two object points at a fixed intensity difference $\alpha=0.6$ for variety values of the point separation $Z_{0}$ under incoherent illumination. Figure 3(a) depicts that for object separation $Z_{0}=3$, there result no dip or flat top and hence no resolution of object points with intensity ratio $\alpha=0.6$. However, under the same conditions of the optical system, there is a clear
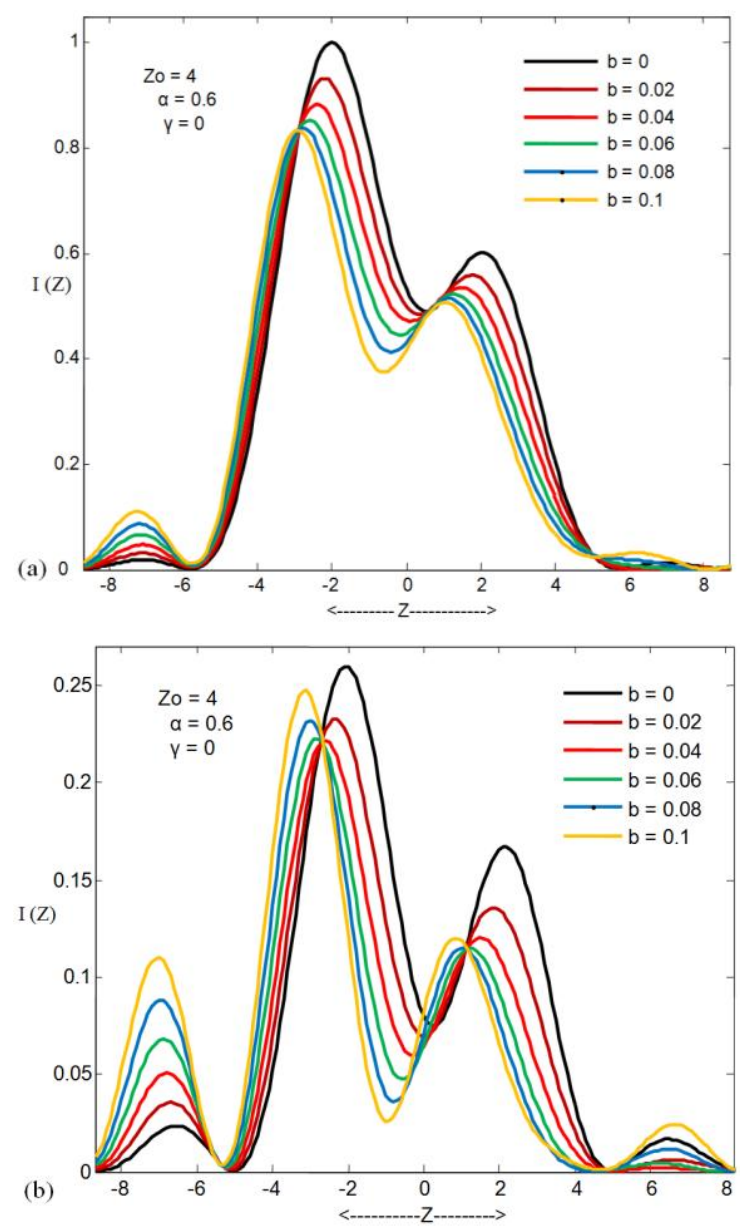

Fig. 2. Profile of the intensity distribution of two object points under incoherent illumination for different widths of semicircular ring over: (a) Transparent central region, (b) superresolver. 

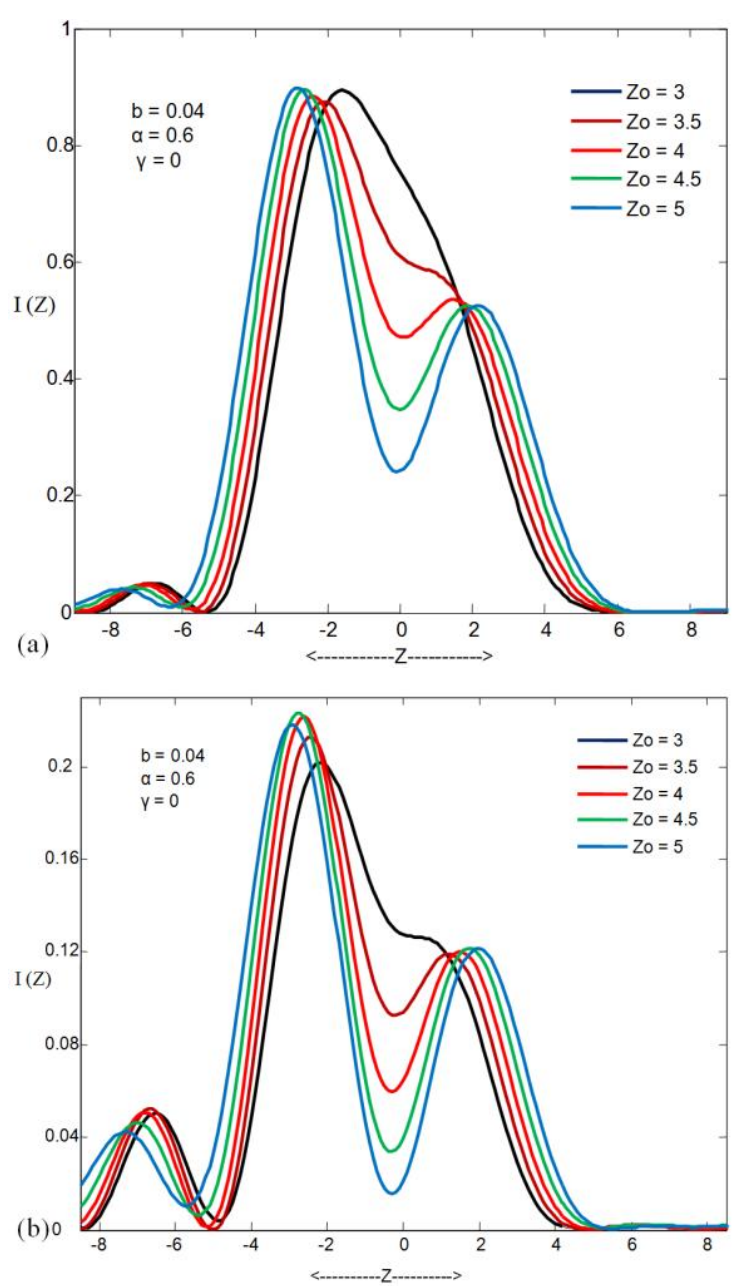

Fig. 3. Image intensity distribution for various values of $Z_{0}$ under incoherent illumination for certain width of semicircular ring over: (a) Transparent circular region, (b) superresolver.

evidence of resolution with the employment of super-resolving filter as noticed in Fig. 3(b). It implies that the super-resolving filter is effective in resolving two point objects with widely varying intensities.

Figure 4 depicts the intensity profile of incoherent composite image of the two-point objects separated by $Z_{0}=3$ with different intensity ratio $\alpha$. Figure 4(a) shows that scarcely any resolution in the two point objects forvarious intensity ratios. In Fig. 4(b) the clear dip indicates that in the case of equally bright object points $(\alpha=1)$, pronounced resolution can be observed than that of the object points with widely varying intensities. The dip in each curve of the intensity profiles in Fig. 4(b)
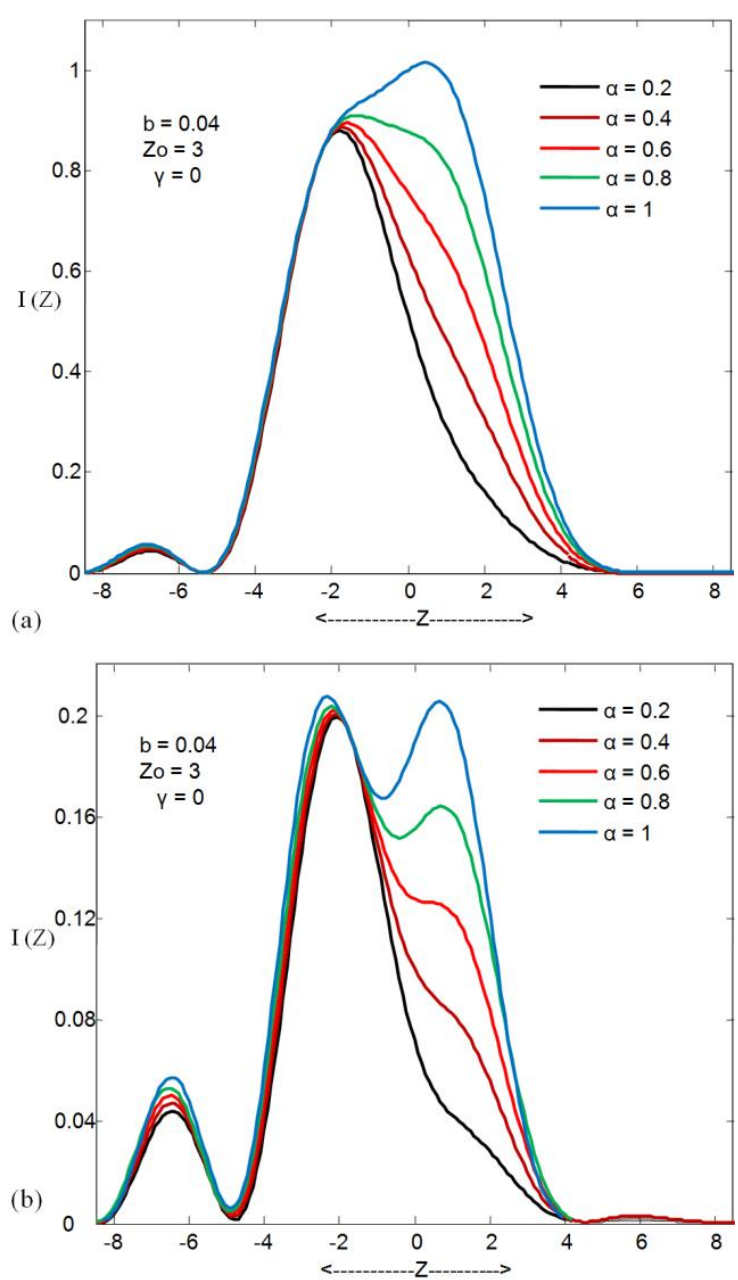

Fig. 4. Image intensity distribution for various values of $\alpha$ in point separation of $Z_{0}=3$ under incoherent illumination for certain width of semicircular ring over: (a) Transparent circular region, (b) superresolver.

indicating the degree of super-resolving nature of the parabolic filter.

Figure 5 shows the intensity profile of composite image of two object points under various degrees of spatial coherent illumination parameter $\gamma$ varying from 0 to 1 . For two point objects separation $Z_{0}=4$, resolution of object points with widely varying intensity, $\alpha=0.6$, are in the neighborhood of being resolved for incoherent illumination, while with increase in coherence parameter, as there is no dip and hence no resolution of the object points under partially coherent and coherent illumination. Figure 5(a) illustrates this feature. However, under similar conditions, the resolving power of the optical system can be enhanced with the use of super-resolving type amplitude filter as can be 

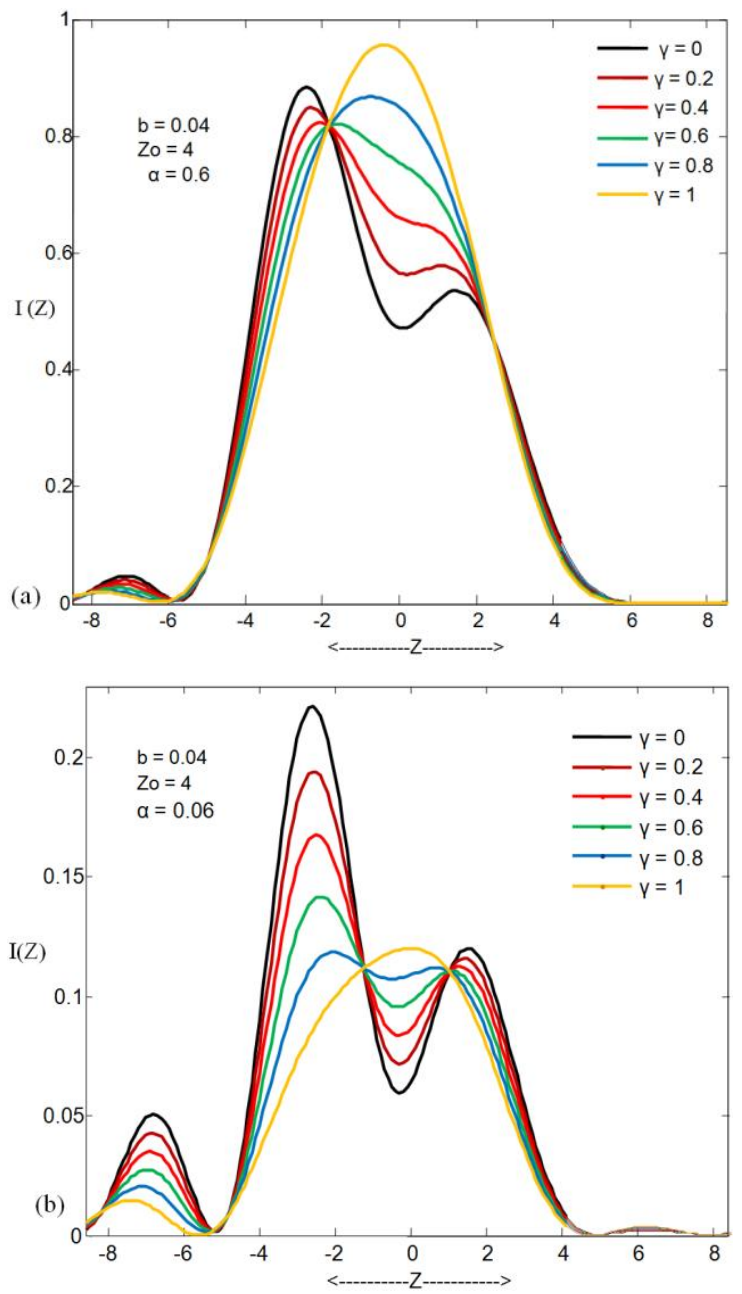

Fig. 5. Image intensity distribution for various values of $\gamma$ in point separation of $Z_{0}=4$ for certain width of semicircular ring over: (a) Transparent circular region, (b) superresolver.

seen from Fig. 5(b).

Variation of sparrow limit with degree of coherence $\gamma$ for unequal and the equally bright object points for different values of semi-circular edge ring width $b$ has been shown in Fig. 6(a) and Fig. 6(b). For $\alpha=0.6$, sparrow limit increases as $b$ increases to 0.2 and then it decreases as $b$ increases to 0.1 . This effect clearly states that overall resolution increases with degree of asymmetric apodization $b$. For any degree of coherence $\gamma$, higher degree of asymmetric apodization $(b=1)$ has lesser limit and hence a higher resolution as compared to Airy pupil as well as other values of $b$. Figure 6(b) reveals that, for equally bright object points resolution limit decreases as $\mathrm{b}$ decreases 0 to 0.04 and then increases for further increase in
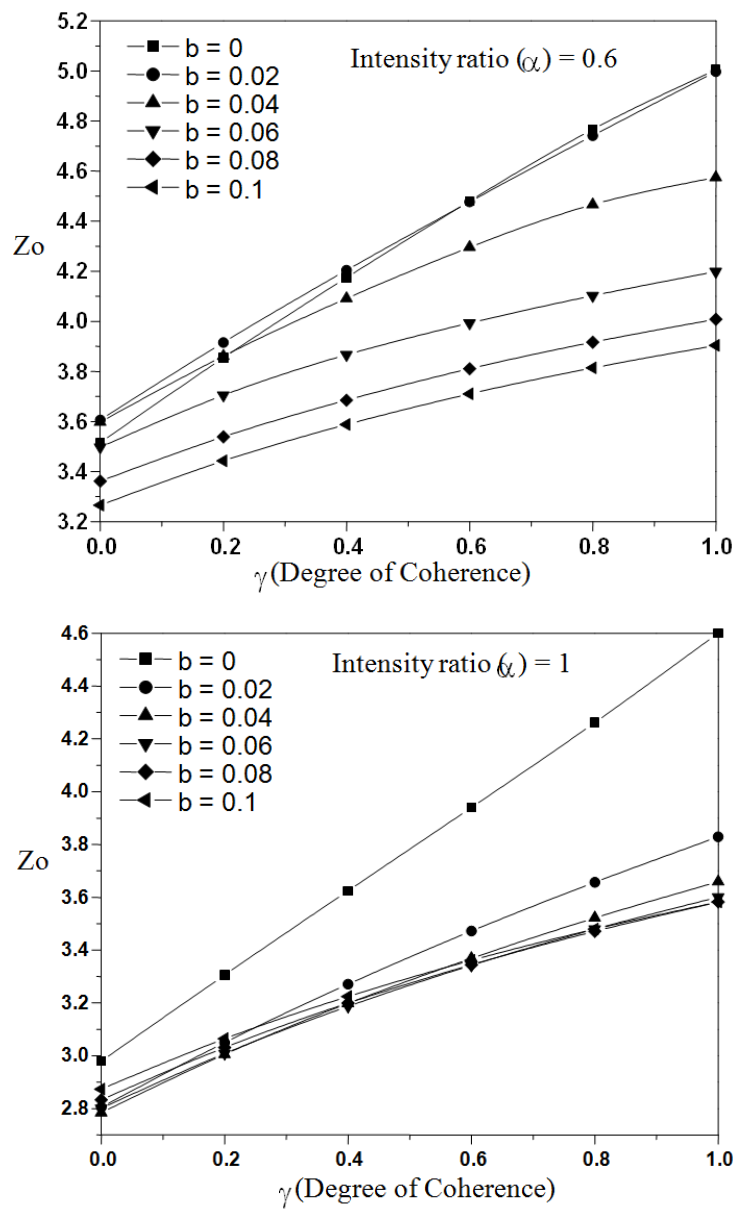

Fig. 6. Sparrow resolution limit $\left(Z_{0}\right)$ as a function of $\gamma$ (degree of coherence) for different values of semi-circular edge ring width $b$ : (a) $\alpha=0.6$ (b) $\alpha=1$.

asymmetric apodization parameter $b$. However, the resolution is high in this case where obtained sparrow limits are lower than that of Airy case for any of degree of asymmetric apodization as $\gamma$ increases 0 to 0.8 . For equally bright object points under coherent illumination, resolution increases to great extent as semi-circular ring width b increases. Figures 7(a) and 7(b) show that, for certain value of semi-circular edge ring width b(0.04 or 0.1$)$ sparrow resolution limit $Z_{0}$ decreases as intensity ratio $(\alpha)$ of two object points increases for any degree of coherence $\gamma$.

\section{Conclusions}

In conclusion we may emphasize that the employment of complex pupil filter in the 

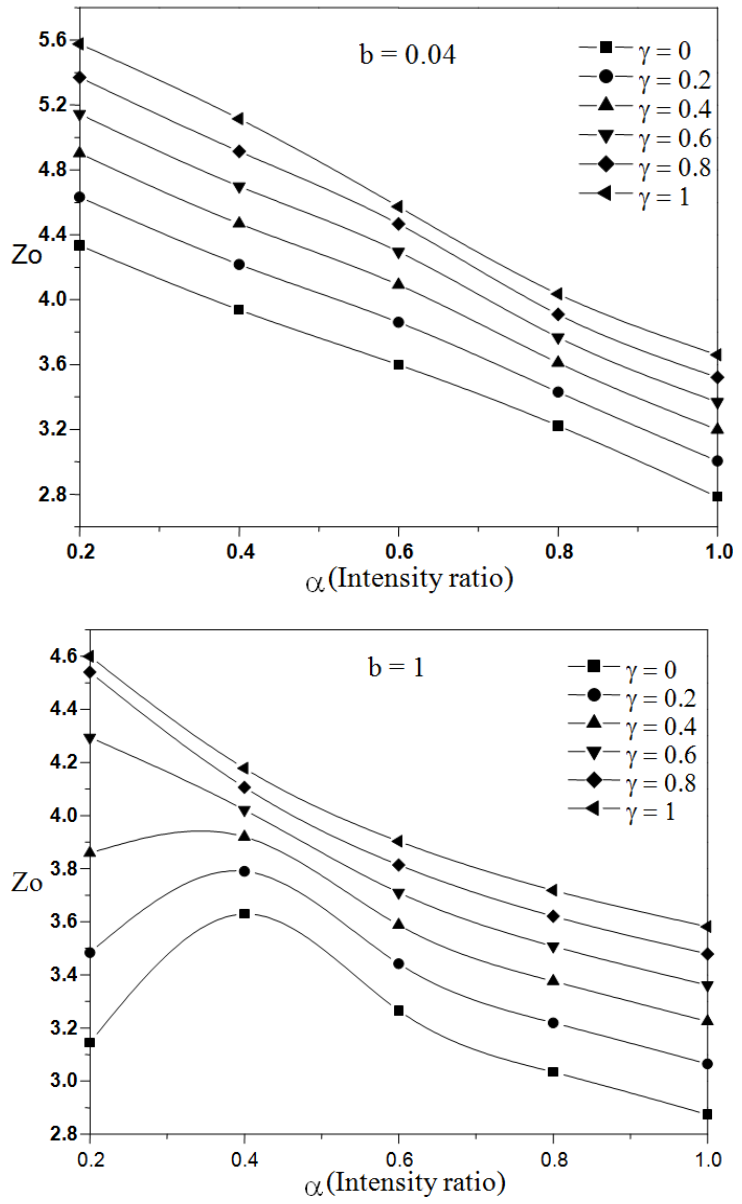

Fig. 7. Sparrow resolution limit $\left(Z_{0}\right)$ as a function of the intensity ratio $(\alpha)$ for various values of $\gamma$ (degree of coherence): (a) $b=0.04$, (b) $b=0.1$.

presence of asymmetric apodisation leads to improved resolution of the optical systems in terms of sparrow limits. The critical Sparrow limit is found to decrease with complex pupil filter when compared to that Airy pupil whatever may be the degree of coherence. This means that the presence of complex pupil functions leads to an improvement in the resolution. For two extreme cases of equally bright object points $(\alpha=1)$, and widely varying intensity ratio $(\alpha=0.2)$, the resolution is pronounced for equally bright object points than that of the other cases. Increase in the degree of coherence lower the resolution with and without modified aperture. Finally, we found that the highest resolution is achieved for superresolving type parabolic filter when equally bright or widely varying intensity object points are illuminated by the incoherent light. On the whole it can be emphasized that an improvement for resolution of two object points with coherent or partially coherent light could be obtained by the use complex pupil filters instead of earlier designed apodizers to perform asymmetric apodization. This would make the design and composition of aperture system little bit complicated but it could be solution for cases in which highly resolved object points are required. Further investigations are being carried out on this subject by considering parameter OTF (Optical Transfer Function) in addition to well defined image quality criterion parameters in next research article communication.

\section{Acknowledgements}

We are grateful to Optics research group, department of physics, University College of science and Nizam College of Osmania University and their contributed publications on two-point resolution work. 